\title{
Giant Cell Tumor of the Distal Tibiofibular Joint: Extensive Curettage, Reconstruction, and Arthrodesis Using Induced Membrane Technique
}

\author{
Sabri Mahjoub ${ }^{1}$, Maamoun Mihoubi ${ }^{2}$, Mourad Zaraa ${ }^{3}$, Liwa Hariz ${ }^{4}$, Mohammed Abdelkefi ${ }^{5}$, Mondher Mbarek ${ }^{6}$
}

\begin{abstract}
Aim: Masquelet technique may be used successfully for the reconstruction and arthrodesis of distal tibiofibular joint after tumor resection. Background: Giant cell tumors (GCTs) involving both distal tibia and fibula are exceedingly rare. The optimal surgical treatment in these locations is controversial. Reconstruction of cavitary bone loss after resection is challenging.

Case description: We describe an unusual presentation of exophytic GCT affecting distal tibiofibular joint in a 35-year-old man. The optimal surgical treatment was controversial. Collapse of the tibial plafond was the major risk of surgical resection. An extensive intralesional curettage was performed. The size of the resulting bone defect measured $5 \mathrm{~cm}$ in fibula and $4 \mathrm{~cm} \times 3 \mathrm{~cm} \times 2 \mathrm{~cm}\left(24 \mathrm{~cm}^{3}\right)$ in tibia. Reconstruction of the cavitary bone loss was challenging. Induced membrane technique was performed for the reconstruction of both tibia and fibula. A plate and a cement spacer were used in the first-time procedure. Two months later, the induced membrane was opened longitudinally. The cement spacer was removed and the biological space which had been created was filled with cancellous bone graft obtained from the patient's iliac crest. Two K-wires were placed in order to avoid tibial plafond collapse and were removed after 6 weeks. Bone union was obtained at 2 months. No recurrence or septic complications were observed. Good functional and anatomic results were obtained at 4 years' follow-up.

Conclusion: Reconstruction and arthrodesis of the distal tibiofibular joint represent an excellent indication of Masquelet technique, especially after GCT resection.

Clinical significance: The present case highlights therapeutic difficulties in this exceptional location, particularly regarding cavitary bone loss. Keywords: Ankle joint, Giant cell tumor of bone, Masquelet technique, Reconstruction, Tumor.

Journal of Foot and Ankle Surgery (Asia Pacific) (2020): 10.5005/jp-journals-10040-1115
\end{abstract}

\section{BACKGROUND}

The giant cell tumor (GCT) of bone is a common benign tumor, locally aggressive and potentially able to metastasize. It arises typically around the knee. Forms involving distal tibia or fibula are exceedingly rare. ${ }^{1}$ The optimal surgical treatment in these locations is controversial. Reconstruction of cavitary bone loss after resection is challenging.

We describe an unusual presentation of exophytic GCT affecting distal tibiofibular joint. Induced membrane technique was performed for the reconstruction of both tibia and fibula. The present case highlights therapeutic difficulties in this rare location which, to the best of our knowledge, has never been reported.

\section{Case Description}

A 35-year-old male presented with persistent left ankle pain and swelling after a mild leg trauma 1 year ago. On physical examination, the left ankle was swollen and tender to touch. However, joint movements were normal.

Plain radiographs showed a well-defined multiloculated and expansile lytic lesion involving both lower ends of tibia and fibula. It had a soap bubble appearance (Fig. 1).

A computed tomography revealed a large lytic lesion damaging tibiofibular joint and more than half of tibial and fibular epiphysis. The tumor had thinned the lateral cortex of the fibula without breaking it. The tibial plafond was thin and there was a serious risk of collapse. Nevertheless, the ankle mortise was preserved (Fig. 2).

Magnetic resonance imaging showed a well-defined heterogeneous lytic lesion. Areas of hemorrhage and necrosis
${ }^{1-6}$ Department of Orthopaedics, Trauma Center, Ben Arous, Tunisia Corresponding Author: Maamoun Mihoubi, Department of Orthopaedics, Trauma Center, Ben Arous, Tunisia, Phone: +216 94731527, e-mail: maamounmihoubi@gmail.com

How to cite this article: Mahjoub S, Mihoubi M, Zaraa M, et al. Giant Cell Tumor of the Distal Tibiofibular Joint: Extensive Curettage, Reconstruction, and Arthrodesis Using Induced Membrane Technique. J Foot Ankle Surg (Asia Pacific) 2020;7(1):24-27.

Source of support: Nil

Conflict of interest: None
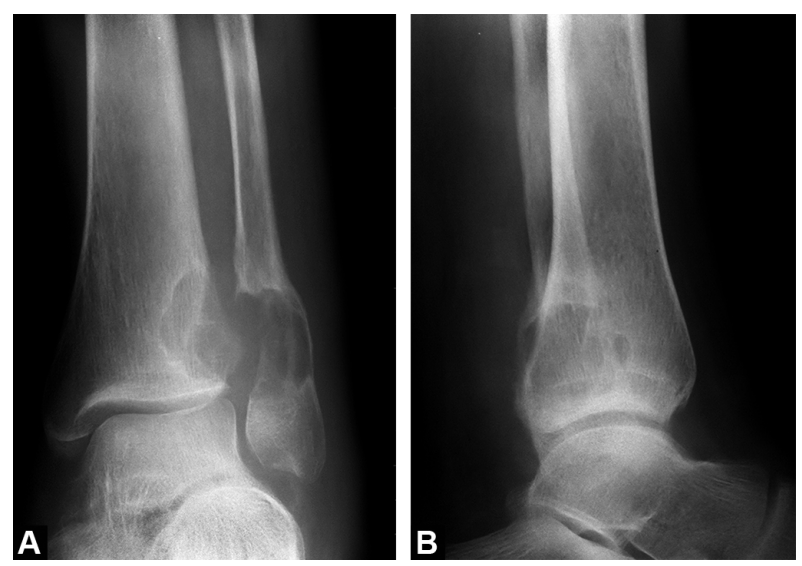

Figs $1 \mathrm{~A}$ and B: Preoperative anteroposterior and lateral radiographies of the left ankle showing a well-defined multiloculated and expansile lytic lesion involving the distal tibiofibular joint 
within the lesion were noted. Minimal posterior soft tissue extension was observed. However, there was no subchondral breakthrough and no extension to the tibiotalar joint (Fig. 3).

All these findings were suggestive of a GCT. Biopsy confirmed the diagnosis. Therefore, we decided to perform extensive curettage, reconstruction, and arthrodesis of the distal tibiofibular joint using the induced membrane technique.

The patient underwent open surgery (Fig. 4). An extensive intralesional curettage was performed. Collapse of the tibial plafond was the major risk of surgical resection. The size of the resulting segmental bone defect in fibula measured $5 \mathrm{~cm}$. The volume of the cavitary bone loss in tibia measured $4 \mathrm{~cm} \times 3 \mathrm{~cm} \times$ $2 \mathrm{~cm}\left(24 \mathrm{~cm}^{3}\right)$. A plate was used to stabilize the ankle mortise. Then a polymethyl methacrylate (PMMA) cement spacer was placed in the bone defect (Fig. 5).

Two months later, the second-time procedure was carried out. The induced membrane was opened longitudinally. The cement spacer was removed. The inner part of the induced membrane located inside the tibia was taken off. Then the biological space which had been created was filled with small morsels of cancellous bone graft obtained from the patient's iliac crest (Fig. 6). Finally,

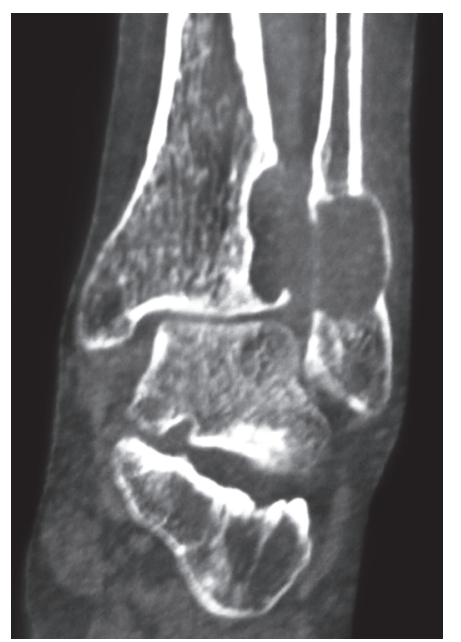

Fig. 2: Coronal CT scan view showing a large lytic lesion damaging the tibiofibular joint

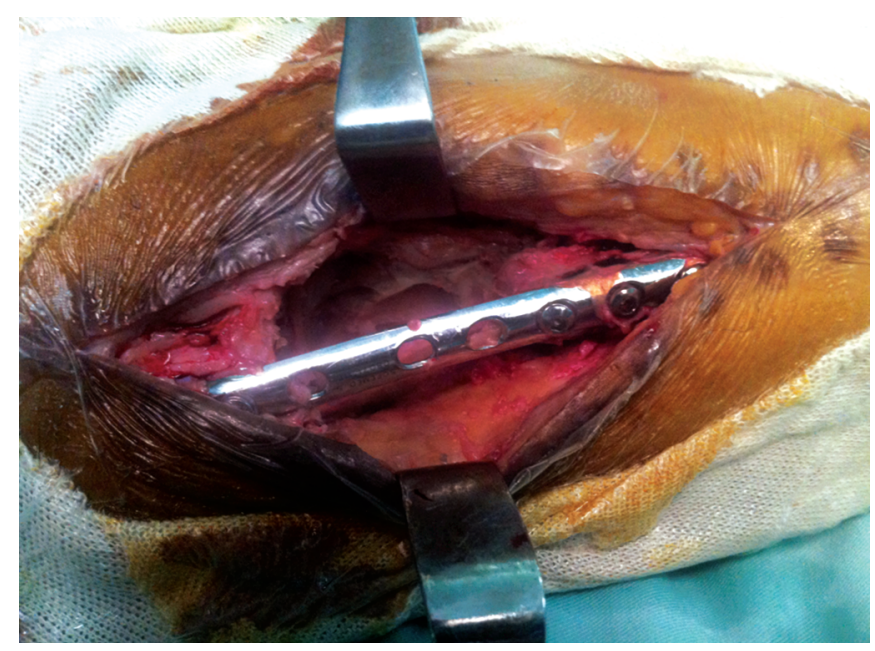

Fig. 4: Intraoperative photography after giant cell tumor excision and stabilization using a plate
K-wires were placed in order to avoid collapse of the tibial plafond (Fig. 7).

Rehabilitation started immediately after operation, and the patient was non-weight-bearing for 6 weeks. Then K-wires were taken away and weight-bearing was gradually increased to full weight-bearing at 3 months. There has been no septic complication or wound dehiscence. Bone union was achieved at 2 months (Fig. 8). At 4 years' follow-up, full and painless range of motion of the ankle was achieved. The hindfoot was well aligned. No local recurrence and no arthritis were detected in imaging studies (Fig. 9).

\section{Discussion}

The GCT of bone is a common benign tumor, locally invasive and potentially able to metastasize. ${ }^{1}$ It is characterized by high local recurrence rates, reaching even up to $65 \%$ of cases. ${ }^{2}$

The GCT of bone arises typically around the knee. Forms involving the ankle are exceedingly rare. In a series of 621 Chinese patients with a GCT of an extremity, Niu et al. ' found only 14 lesions

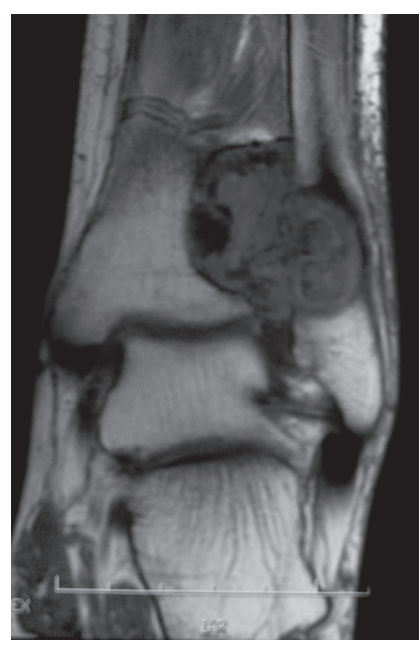

Fig. 3: Coronal MRI view showing a heterogeneous lytic lesion with no subchondral breakthrough and no extension to the tibiotalar joint

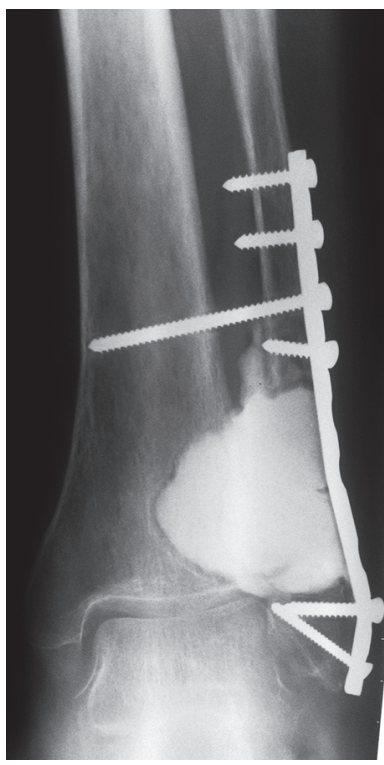

Fig. 5: Immediate postoperative anteroposterior radiography of the left ankle, showing the bone defect filled with acrylic cement 


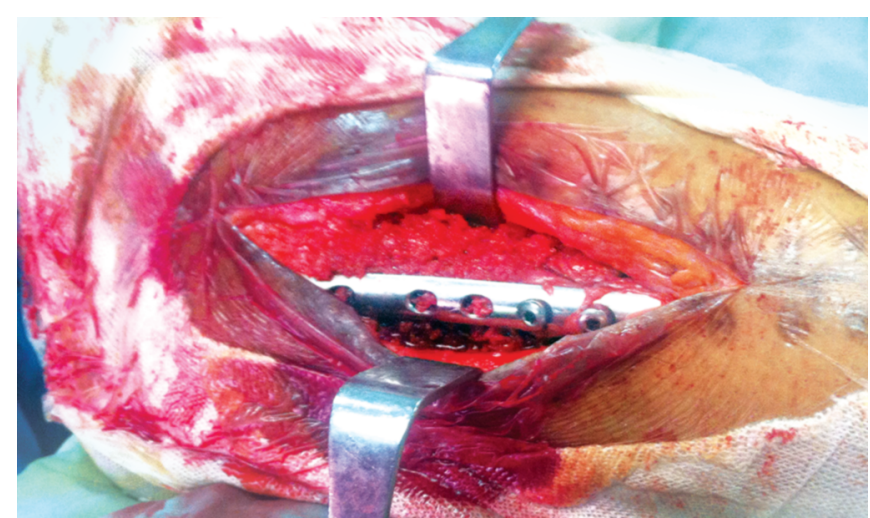

Fig. 6: Intraoperative photography showing spongy autologous bone graft after removal of the cement

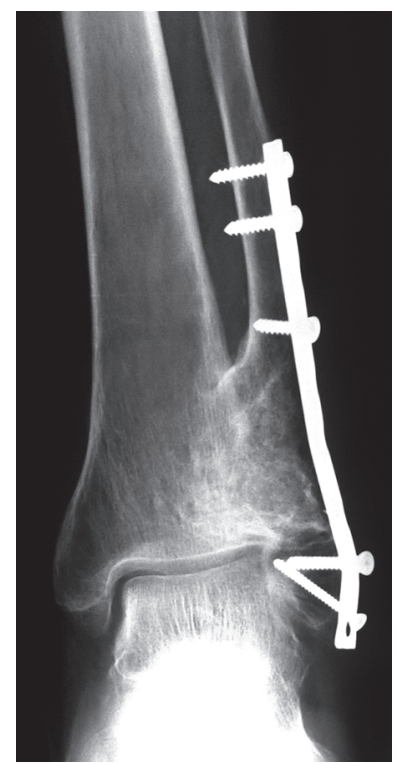

Fig. 8: Anteroposterior radiography of the left ankle showing the bone consolidation and the absence of tumoral recurrence

located in the lower end of tibia and only one case of distal fibular GCT. To the best of our knowledge, GCT affecting simultaneously distal tibia and fibula has never been reported.

Surgical options are controversial in these rare locations. While intralesional curettage is associated with high recurrence rates, en bloc resection provides better removal of the tumor. ${ }^{2}$

En bloc resection of the lower end of tibia requires tibiotalar arthrodesis, which is associated with high morbidity. Casadei et al. reported several complications after ankle arthrodesis, using a transplantar intramedullary nail or a plate. ${ }^{3}$ Otherwise, total ankle arthroplasty may be suitable in case of recurrent and aggressive tumor over the distal tibia. ${ }^{4}$

En bloc resection of distal fibula has been reported by several authors without requiring ankle arthrodesis. Jones et al., ${ }^{5}$ in a cadaver study, demonstrated that distal fibular resection caused an increase in talar tilt and anterior drawer. These parameters decreased significantly with ligament repair. Based on these anatomical results, Monson et al. ${ }^{6}$ described an innovative surgical technique for lateral ankle stabilization after distal fibular resection using the peroneus brevis tendon. Nadkarni et al. ${ }^{7}$ described another technique using ipsilateral proximal fibula which was reversed and fixed to distal tibia.

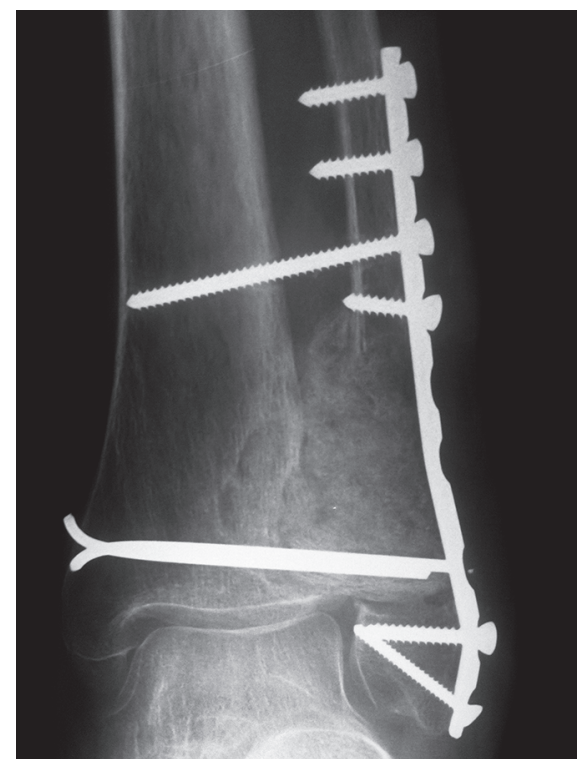

Fig. 7: Immediate postoperative anteroposterior radiography of the left ankle after second stage procedure of Masquelet technique

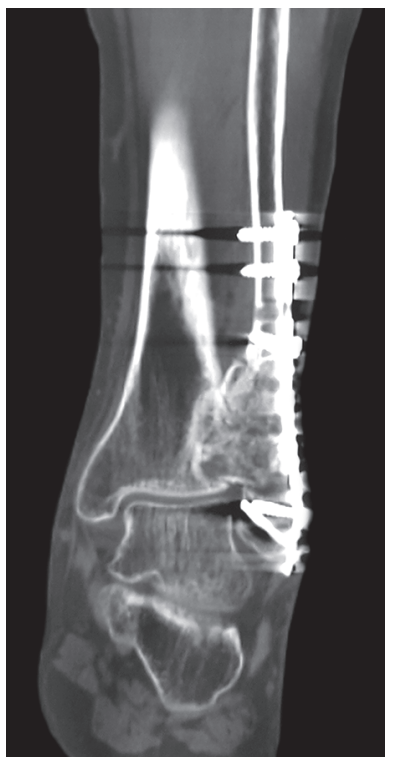

Fig. 9: CT scan view showing the consolidation and the absence of tumoral recurrence at four years followup

Wide resection is known to produce low recurrence rates. Still these techniques were associated with high complication rates. Some authors suggested the use of local adjuvants such as PMMA after intralesional curettage, which decreased morbidity and local recurrence. $^{2}$

Polymethyl methacrylate is thought to improve local control through its thermal and toxic effects against tumoral cells. In a systematic review, Zuo et al. ${ }^{8}$ reported lower recurrence rates in patients treated with PMMA cementation following intralesional curettage compared to those treated with bone grafting.

Several authors described extensive curettage which is performed through a cortical window, generally a thinned or destroyed cortex. All gross tumor tissues are exposed and curetted. Safe margins of resection are obtained, except on the articular side where subchondral bone is maintained. This technique produced low recurrence rates, dropping down to $8.6 \%{ }^{1}$ 
In the present case, we performed extensive curettage in order to reduce morbidity and to control recurrence. We paid attention to preserve the ankle mortise. Then we filled the residual cavity with bone cement (PMMA) with a dual objective of destroying tumoral cells and inducing a surrounding biological membrane.

Based on the general concept of the tissular response to an enclosed foreign body, the induced membrane technique, the so-called Masquelet technique, comprises two surgical phases. First, a cement spacer is placed into the bone defect. Then a few weeks later, the cement is taken away and the cavity is packed with cancellous bone graft. This technique has been used for treatment of various bone defects resulting from different etiologies. Nonetheless, poor experience was reported with the fibula. ${ }^{9}$ To the best of our knowledge, this technique has never been described for the reconstruction of bone defects following distal tibia or fibula resection for GCT.

The present case highlights therapeutic difficulties in this exceptional location, particularly regarding cavitary bone loss. In this case, the surrounding induced membrane may interfere with fusion after bone grafting. Thus, the part of the membrane located inside the recipient bone should be removed. ${ }^{9}$

As a final point, distal tibiofibular arthrodesis is inevitable in this case. Good functional results were obtained in a similar case of distal tibiofibular arthrodesis for osteochondroma in the fibula. ${ }^{10}$

\section{Conclusion}

Masquelet technique, also called induced membrane technique, may be used successfully for the reconstruction and arthrodesis of distal tibiofibular joint following tumor resection, as in our case.

\section{Clinical Significance}

There are major therapeutic difficulties of giant cell tumor located in distal tibiofibular joint, particularly regarding cavitary bone loss.

\section{REFERENCES}

1. Niu X, Zhang $Q$, Hao L, et al. Giant cell tumor of the extremity. retrospective analysis of 621 chinese patients from one institution. J Bone Joint Surg Am 2012;94(5):461-467. DOI: 10.2106/JBJS.J.01922.

2. Klenke FM, Wenger DE, Inwards CY, et al. Giant cell tumor of bone. risk factors for recurence. Clin Orthop Relat Res 2011;469(2):591-599. DOI: 10.1007/s11999-010-1501-7.

3. Casadei R, Ruggieri P, Giuseppe T, et al. Ankle resection arthrodesis in patients with bone tumors. Foot Ankle Int 1994;15(5):242-249. DOI: 10.1177/107110079401500503.

4. Ajit Singh V, Nasirudin N, Bernatt M. Endoprosthetic reconstruction for giant cell tumors of the distal tibia: a short term review. Asia-Pac J Clin Oncol 2013;9(2):182-189. DOI: 10.1111/j.1743-7563.2012.01553.x.

5. Jones RB, Ishikawa SN, Richardson EG, et al. Effect of distal fibular resection on ankle laxity. Foot Ankle Int 2001;22(7):590-593. DOI: 10.1177/107110070102200711.

6. Monson DK, Vojdani S, Dean TJ, et al. Lateral ankle stabilization after distal fibular resection using a innovative approach: a surgical technique. Clin Orthop Relat Res 2014;472(4):1262-1270. DOI: 10.1007/ s11999-013-3408-6.

7. Nadkarni S, Punit AS, Nair RV. Giant cell tumour of distal fibula managed by en block resection and reconstruction with ipsilateral proximal fibula. J Orthop Case Rep 2015;5(1):52-54. DOI: 10.13107/ jocr.2250-0685.255.

8. Zuo D, Zheng L, Sun W, et al. Contemporary adjuvant polymethyl methacrylate cementation optimally limits recurrence in primary giant cell tumor of bone patients compared to bone grafting: a systematic review and meta-analysis. World J Surg Oncol 2013;11(1):156. DOI: 10.1186/1477-7819-11-156.

9. Bégué T, Auregan JC. The induced membrane technique for treatment of large segmental or cavitary bone defects: How to maximize the success. Arch Ortop Reumatol 2013;124(1-3):18-19. DOI: 10.1007/ s10261-013-0046-1.

10. Sorensen BW, Mikkelsen P. Arthrodesis of the distal tibiofibular joint for an osteochondroma in the fibula Encroaching on the distal tibia and involving the talocrural joint: a case report. J Foot Ankle Surg 2012;51(5):664-665. DOI: 10.1053/j.jfas.2012.05.014. 